\title{
LA COMISIÓN NACIONAL DE BIOÉTICA DE CHILE: UNA TA- REA PENDIENTE. APORTES DE LA EXPERIENCIA DE LAS CO- MISIONES NACIONALES DE BIOÉTICA DE MÉXICO E ITALIA
}

Paulina Ramos Vergara ${ }^{1}$, Ángela Arenas Massa ${ }^{1}$, Manuel J. Santos ${ }^{2}$

Resumen: El objetivo de este trabajo es discurrir sobre los orígenes, naturaleza, integración y funciones de las Comisiones Nacionales de Bioética de México e Italia, con el fin de extraer experiencia que pudiese ayudar al momento de implementar la Comisión Nacional de Bioética de Chile. La investigación es cualitativa-interpretativa, fenomenológica, descriptiva y bibliográfica. Se infiere que la Comisión Nacional Bioética de Chile, para cumplir la función encomendada, debiese considerar la experiencia de otras comisiones, en particular la de México e Italia.

Palabras clave: Comisión Nacional de Bioética Chile, CONBIOÉTICA, Comitato Nazionale di Bioetica

The Bioethics National Commission in Chile: a pending task. Contributions from the experience of national bioethics commissions of Mexico and Italy

\begin{abstract}
The aim of this study is to reflect on the origin, nature, integration and functions of National Bioethics Commissions of Mexico and Italy, with the goal to draw helpful experience for implementing the National Bioethics Commission in Chile. The study is qualitative-interpretative, phenomenological, descriptive and bibliographical. It is inferred that the National Bioethics Commission in Chile, in order to fulfill the function entrusted, it must consider the experience of other commissions, in particular that of Mexico and Italy.
\end{abstract}

Key words: National Bioethics Commission in Chile, CONBIOÉTICA, Comitato Nazionale di Bioetica

A Comissão Nacional de Bioética do Chile: uma tarefa pendente. Contribuiçóes da experiência das comissóes nacionais de bioética do México e da Itália

Resumo: O objetivo deste trabalho é discorrer sobre as orígens, natureza, integração e funçôes das Comissões Nacionais de Bioética do México e da Itália, com a finalidade de obter experiência que possa ajudar na ocasião de implementar a Comissão Nacional de Bioética do Chile. A investigaçáo é qualitativa-interpretativa, fenomenológica, descritiva e bibliográfica. Infere-se que a Comissão Nacional Bioética do Chile, para cumprir a função para a qual foi encarregada, deveria considerar a experiência de outras comissóes, em particular a do México e da Itália.

Palavras-chave: Comissão Nacional de Bioética do Chile, CONBIOÉTICA, Comitato Nazionale di Bioetica

\footnotetext{
${ }^{1}$ Centro de Bioética. Facultad de Medicina Pontificia Universidad Católica de Chile, Chile Correspondencia: pramosv@med.puc.cl

${ }^{2}$ Facultades de Ciencias Biológicas y Medicina, Centro de Bioética, Pontificia Universidad Católica de Chile, Chile
} 


\section{Introducción}

"El cambio social está enraizado en los esfuerzos conscientes de las personas para resolver problemas mutuos a través de acciones colectivas". Máximo Pacheco(1:516).

Las Comisiones Nacionales de Bioética son recomendadas por Unesco(2) como instancias independientes, pluridisciplinarias, pluralistas(3) y consultivas. México creó la Comisión Nacional Bioética por Decreto Supremo No 7, Diario Ofcial 7 septiembre 2005(4); Italia crea el Comitato Nazionale Bioetica, por Decreto Presidente Consiglio Ministri, 28 marzo 1990(5); Chile, por Ley No 20.120, publicada en el Diario Oficial el 22 de septiembre de 2006(6), instituye la Comisión Nacional de Bioética; a la fecha, el nombramiento de sus miembros está pendiente y, por ende, también la entrada en ejercicio de sus funciones.

Las Comisiones Nacionales de Bioética no responden a un modelo único. Algunas tienen un carácter permanente, por ejemplo, Francia(7), Italia, Argentina, México; otras se crean ad hoc para el estudio de un tema específico, entre estas las de Estados Unidos de Norteamérica(8) y en el Reino Unido. Asimismo, difieren en su nominación ("Comisión, "Comité", "Consejo"), composición, organización, dependencia y funciones.

La Declaración de Helsinki (Revisión de Tokio, 1975) hace referencia a estas comisiones como responsables de supervisar o aconsejar a los comités de ética, protectores de una deontología médica, (y también) de ser testimonios de una ética universal, una visión generalmente aceptada de la dignidad de la vida humana y de los límites que no pueden ser franqueados si no se quiere violar esta dignidad.

Algunos estiman que las comisiones nacionales de bioética deben promover un cambio social, una cultura ética, a través de su autoridad fundada en el prestigio de sus decisiones, ya que si estas no son comprendidas, resguardadas y aplicadas por la misma sociedad, quedarán en el vacío aplicativo $y$, por tanto, en la invisibilidad e inoperatividad de esta instancia(9). Estas comisiones, según Cortina(10), deben configurar: "Un mínimo de acuerdos morales que traspasen las fronteras y forjen una ética cívica transnacional”.
A la fecha, además de Argentina ${ }^{3}$, Chile y México, varios otros países de Latinoamérica han creado comisiones de bioética nacionales, a través de algún órgano del Estado, entre esas: Bolivia, Brasil, Colombia, Ecuador, El Salvador, Haití y Venezuela(11).

La Unesco distingue tres tipos de comisiones, a saber:

- Comisiones establecidas por resolución de un órgano gubernamental, en la mayoría de los casos el Parlamento o el ministro competente (México, Italia y Chile). En otros casos, pueden ser establecidas por el presidente o por el gobierno de un Estado (Venezuela). Según su tipo de gestación serán los poderes atribuidos, ya que son el resultado de una decisión política nacional.

- Comisiones establecidas por un órgano nogubernamental, como por ejemplo una organización profesional (academia de ciencias o asociación médica) o un órgano consultivo (consejo de la salud), o por una ONG (República Dominicana).

- Comisiones creadas por un Estado miembro para el efecto de tener representación en la Unesco.

La Comisión Nacional de Bioética México, en adelante CONBIOÉTICA, tiene una experiencia de ocho años de funcionamiento. Por su parte, el Comitato Nazionale per la Bioetica italiano, en adelante CNBI, de 23 ańos. El criterio de inclusión comparativa de este trabajo es que México e Italia constituyen experiencias prestigiosas en sus respectivos continentes, fueron creadas por resolución de un órgano gubernamental y son países de origen latino, por lo que son adecuadas para que sus experiencias puedan ser consideradas por la Comisión Nacional de Bioética de Chile, en adelante CBN Chile.

\footnotetext{
${ }^{3}$ Resolución No 666, Ministerio de Justicia Argentina (13.05.2010). Resolución No 126 de 2003, Ministerio de Salud y Previsión Social Bolivia , (27.03. 2003); Resolución Presidencial Nº196 de 1996, Presidencia Brasil 1996, (10.10.1996); Ley N 1374 de 2010, Parlamento Colombia (08.01.2010); Resolución N²110 de 2012, Ministerio de Salud Pública Ecuador, (11.10.2012); Resolución №212 de 2009, Ministerio de Salud El Salvador (21.10.2009). Resolución $\mathrm{N}^{\circ} 99$ de 1999, Ministerio de Salud Haití (23.12.1999). Decreto $\mathrm{N}^{\circ}$ 7666 de 2010, Presidencia de la República Venezuela (13.09.2010).
} 


\section{Desarrollo}

1. Comisión Nacional de Bioética de México y Comitato Nazionale per la Bioetica Italiano

a) En cuanto a su origen y naturaleza. CONBIOÉTICA es un órgano creado por Decreto Supremo $\mathrm{N}^{\circ}$ 7, (DOF), (publicado Diario Oficial 07.09.2005), asesor de los Poderes del Estado, que goza de autonomía técnica y operativa. Dado que México es un Estado federal, además de la Comisión Nacional de Bioética se crearon comisiones estatales.

Respecto de Italia, la Comisión es un órgano colegiado, creado por Decreto del Presidente Consiglio Ministri, 28 marzo de 1990, que recibe Recomendaciones de la Asamblea Parlamentaria del Consejo de Europa, en particular, la Recomendación No 1.100, de 2 febrero de 1989, la Resolución del Parlamento Europeo de 13 febrero de 1989 y la Resolución No 6-00038 de 5 julio de 1988 de la Cámara de Diputados, que le solicita el cometido. Se trata de un órgano dependiente de la Presidenza del Consiglio dei Ministri, con autonomía técnica, cuyas función es absorver las consultas sobre la materia formuladas por el gobierno, operadores normativos de la administración del Estado, Parlamento, comités regionales de bioética y otras instituciones (los comités regionales no dependen del CNBI). Además, la CNBI puede activar iniciativas propias o en asociación con las siguientes instituciones: ministerios y sus órganos descentralizados, entidades locales (región, provincia o municipios), universidades, escuelas y liceos, organizaciones nacionales, internacionales, academias, asociaciones, comités de ética nacionales y extranjeros, entidades y fundaciones de investigación y promoción cultural —en la medida que sean reconocidas por el Estado italiano-, y colegios profesionales legalmente constituidos(12).

b) En cuanto a su integración. CONBIOÉTICA cuenta con un consejo; un director ejecutivo y unidades administrativas. El consejo lo integra seis consejeros y un presidente, designados por el secretario de salud. Este además puede invitar a participar en el consejo a distinguidas personalidades de la sociedad civil y de la comunidad médica, quienes solo tienen derecho a voz.
A excepción del presidente del Consejo, a los designados como consejeros se les reconoce un carácter honorífico, duran en su cargo cuatro años y, a excepción del presidente, no pueden ser ratificados para periodos posteriores. Los consejeros pueden designar a sus respectivos suplentes para casos de ausencias. El consejo sesiona ordinariamente por lo menos cada dos meses y de manera extraordinaria cuando la urgencia de algún asunto así lo requiere.

El CNBI está constituido por un presidente, vicepresidentes, consejo de la presidencia y asamblea. Para el desarrollo de sus actividades estructura grupos de trabajo, con la eventual participación consultiva de expertos. También forman parte del CNBI los presidentes honorarios, es decir, los miembros permanentes investidos como máxima autoridad en periodos precedentes, o personalidades tan destacadas como un premio Nobel de Medicina(13).

El consejo de la presidencia está compuesto por el presidente del CNBI y los vicepresidentes; mantiene reuniones periódicas con los coordinadores de los grupos de trabajo.

La asamblea está compuesta por el presidente, los vicepresidentes, presidentes honorarios y miembros nominados al efecto (generalmente provenientes del mundo académico), y su número varía según la investidura del cargo (han aumentado los presidentes honorarios, por ejemplo); no obstante, la conformación varía entre 44 y 45 miembros.

Los miembros del CNBI son nombrados por periodos no definidos estrictamente; en las primeras dos nominaciones se fija un periodo de dos años, la tercera y cuarta nominación aumenta a tres años, la quinta nominación de cuatro años y la actual nominación (2006-2013) de siete años.

El presidente, los presidentes honorarios, los vicepresidentes y los miembros del CNBI no reciben remuneración por el ejercicio de sus funciones; solo tienen derecho al reembolso de los gastos que les irrogan sus responsabilidades, con categoría equivalente a primer grado del escalafón en la administración pública.

c) En cuanto sus funciones. Las tareas más destaca- 
das de la CONBIOÉTICA son: establecer las políticas públicas de salud vinculadas con la temática bioética; servir como órgano de consulta nacional sobre temas específicos de bioética; identificar y sistematizar los elementos que inciden en una cuestión bioética, con el fin de ofrecer información pertinente sobre los mismos a instituciones, grupos sociales o cualquier otro sector interesado; coadyuvar para que el derecho a la protección de la salud se haga efectivo en los temas de investigación para la salud, así como en la calidad de la atención médica; propiciar debates sobre cuestiones bioéticas con la participación de los diversos sectores de la sociedad; fomentar la enseńanza de la bioética (atención médica e investigación); opinar sobre los protocolos de investigación en salud que se sometan a su consideración; suscribir los convenios de colaboración que sean necesarios para el cumplimiento de su objeto; fomentar la comunicación con el mundo universitario y la sociedad civil en cuestiones bioéticas (seguridad, entre otros).

Por su parte, las funciones más importantes de CNBI son: elaborar (previo conocimiento y apoyo de instancias nacionales y/o internacionales pertinentes) un resumen de los programas, objetivos y resultados de la investigación y experimentación en el campo de las ciencias de la vida y de la salud del hombre; formular opiniones "pareri" (que siempre se deben iniciar con el status quaestionis) y proponer soluciones -incluyendo el fin de asesorar procesos legislativos- para hacer frente a problemas éticos y jurídicos emergentes en investigación y posibles aplicaciones clínicas en en resguardo de la dignidad, derechos humanos y otros valores a los que Italia se adhiere, a través de la Carta Constitucional e instrumentos internacionales; plantear soluciones en las funciones de control dirigidas a la tutela de la seguridad del hombre y del ambiente en la producción de material biológico; recomendar medidas de protección de los eventuales riesgos en los pacientes tratados con productos fruto de ingeniería genética o sometidos a terapia génica; promover la redacción de códigos deontológicos para operadores de los varios sectores interesados, y promover una correcta información de la opinión pública.

El reglamento interno del CNBI, aprobado en asamblea plenaria en el año 2008 (después de 18 años de experiencia y funcionamiento), indica que los documentos emitidos por el órgano colegiado son de tres tipos: opiniones ("pareri”), aprobadas por la asamblea (quórum de mayoría absoluta de miembros en sala y se requiere la mitad de los miembros en ejercicio para sesionar); mociones ("mozioni"), informes emitidos con carácter de urgente, aprobados por $2 / 3$ de los presentes en la asamblea, y respuestas ("risposte") a cuestiones requeridas por otras entidades o personas físicas al CNBI. Esta comisión, en forma excepcional, se pronuncia sobre planteamientos formulados por personas individuales y ello solo cuando está involucrado el interés general(14).

d) En cuanto a las observaciones. Para CONBIOÉTICA se advierte, en lo referente a su integración, que los miembros son elegidos únicamente por el secretario de salud. Sobre el nombramiento de los suplentes, solo los comisionados están facultados para designarlos. Además, quien ejerce el cargo de presidente puede ser reelecto sin límite de años. En el consejo de la comisión pueden participar personalidades de la sociedad civil y médica con solo derecho a voz, en la medida que sean invitadas por el secretario de estado. Es probable que en una sociedad ideologizada no sean invitados los más críticos ni los posibles opositores al gobierno de turno.

Para CNBI se advierte, en lo referente a su integración, la preocupación del Estado de garantizar la pluralidad de los miembros que la integran; se exige a los grupos de trabajo mantener en su quehacer parámetros técnicos propios de una cultura bioética, sensible a las señales de la sociedad y que conformen un espacio de reflexión orgánica pluridisciplinar. Y a los grupos de trabajo se les garantiza la libertad constitucional de "ciencia y enseñanza”, de acuerdo con el artículo 33 de la Constitución italiana, y libertad de expresión (artículo 21 del mismo cuerpo normativo $)^{4}$.

\footnotetext{
${ }^{4}$ CONBIOÉTICA, La Clonación Humana, Revista Debate Bioético en la ONU. (2007); Edición y difusión de la Guía Nacional para la integración y funcionamiento de Comités de Ética en Investigación (2009); Edición y difusión de la Guía Nacional para la integración y funcionamiento de Comités de Hospitalarios de Bioética (2009); Edición y difusión del documento Evaluación Operacional CEBs (2010). Disponible en www.conbioeticamexico.salud.gob. $\underline{\mathrm{mx} / \text { descargas/pdf/publicaciones/revistadebate/clonacionhumana. }}$ pdf (30.04.2013).
} 
En cuanto a los periodos de los miembros en el ejercicio de sus funciones, llama la atención la irregularidad en la CNBI; ella se explica por las viscisitudes políticas de los últimos años (en dos ocasiones el quinto periodo ha sido prorrogado por decreto).

De las funciones de CONBIOÉTICA, sorprende que su ámbito de acción sea excesivamente amplio y a la vez heterogéneo, haciendo difícil responder a dichas demandas con estándares de calidad. Cumple funciones consultivas en bioética; diseña políticas públicas; promueve la enseñanza, capacitación y discusión en bioética(15); consolida la infraestructura bioética en el país con la creación de comisiones estatales de bioética (CEBs); comités hospitalarios de bioética (CHBs); comités de ética en investigación (CEIs); participa en el debate bioético de orden internacional(16) y además se considera que debe asumir "las demás atribuciones que le asigne el Secretario de Salud".

Entre sus funciones permanentes, sobresale la organización de eventos académicos, para abrir espacios de intercambio y comunicación entre profesionales interesados en contribuir al conocimiento y la práctica bioética.

Por su parte, las funciones de la CNBI demuestran la pertinencia de su delimitación técnica, entre otras cosas por su gran aporte en los ámbitos nacional e internacional. Se pueden enunciar, por ejemplo, Forum of International Ethics, encuentro con los comités de Europa - NEC Forum-, International Dialogues on Bioethics (encuentro con los comités del mundo), European Group on Ethics in Science and New Tecnologies (EGE), Consejo de Europa, a través del Comité Internacional de Bioética (CIB) y el Comité Intergubernativo de Bioética (CIGB), simposios internacionales, en los que los Estados de la Unión Europea se ponen al servicio de ella, y presentación traducida de toda su documentación en inglés a través de su página web(17).

\section{Comisión Nacional de Bioética de Chile}

a) En cuanto a su origen y naturaleza. En Chile, la Comisión Nacional de Bioética (CNB) es de reciente data. Se crea por Ley N ${ }^{\circ} 20.120$, de 22 de septiembre de 2006, y fija normas sobre investigación científica en seres humanos, legisla sobre el genoma humano y prohíbe la clonación humana. Solo los artículos 15 y 16 de dicha ley regulan la mencionada Comisión. Dicha ley se originó por moción parlamentaria de los honorables senadores Mariano Ruiz-Esquide, Nicolás Díaz, Juan Hamilton, Sergio Paez y Andrés Zaldivar, y hace mención a la clonación de mamíferos superiores, lo que ha conmovido al mundo "tanto por la espectacularidad de lo obtenido en la manipulación genética, como por sus eventuales aplicaciones en el ser humano". Agregan: "Sin embargo, estos logros científicos no son los únicos que abren predicciones alarmantes en el campo ético y por ello es necesario enmarcarlas en el amplio campo de la investigación científica en los seres humanos, ámbito en el cual no existe un marco de conductas que concilie el derecho a la investigación y la libertad que una sociedad moderna debe garantizar, y las regulaciones bioéticas que dan cuenta del consenso mayoritario existente hoy en el mundo en este aspecto" (18).

En el art. 14 de la Ley referida se crea la comisión asesora del Presidente de la República denominada "Comisión Nacional de Bioética".

b) En cuanto a su integración. Este tema fue uno de los más debatidos. Durante el proceso legislativo, el proyecto de ley sufrió varios cambios en lo rido a la integración y funciones de la Comisión Nacional de Bioética. Llama la atención la integración y designación de los miembros que el proyecto de ley original contemplaba: un académico especializado en bioética, designado por los decanos de las facultades de Medicina de las universidades reconocidas por el Estado; un académico especializado en bioética, designado por los decanos de las facultades de Derecho de las universidades reconocidas por el Estado; un académico especializado en ética, designado por los decanos de las facultades de Filosofía de las universidades reconocidas por el Estado; el presidente de la comisión de ética del Colegio Médico más representativo de Chile o la persona que él designe; un teólogo designado por el conjunto de las iglesias de Chile; un representante de las sociedades o corporaciones científicas relacionadas directamente con la bioética y un representante del Programa Regional de Bioética de la Organización Panamericana de la Salud, mientras este programa tenga su oficina en Chile. 
La Comisión de Salud del Senado propuso, en cambio, que la integraran cuatro académicos, designados por el Consejo de Rectores, que pertenecieran a las Facultades de Medicina, Derecho, Ciencias y Filosofía de las universidades que lo integran; tres personas designadas por el Instituto de Chile y que formen parte de las Academias de Medicina, de Ciencias y de Ciencias Sociales, Políticas y Morales; una persona designada por el Senado y una nombrada por el presidente de la República (Informe comisión de salud del senado de fecha 20.07.2004, boletín 1993-11). La historia de la ley no deja constancia de los argumentos para variar la integración de esta Comisión $\mathrm{Na}$ cional.

Se entiende polémica la designación e integración, porque se se espera que sea integrada por especialistas en bioética del más alto nivel y que sean representativos de diversas sensibilidades nacionales. En similares términos se manifiesta el Dr. Kottow, al sostener que la comisión debe darse "...en una democracia pluralista, donde sea posible representar opiniones diversas".

El legislador nacional buscó reflejar en la integración de la comisión el carácter interdisciplinar y pluricultural, esto es, la participación de profesionales de diversas ramas científicas y con representantes de entidades de diversidad cultural y expertos en bioética(19).

Los miembros de la Comisión Nacional duran cuatro años en sus cargos, pudiendo ser reelegidos. Se observa que, con la reelección sin límites, los miembros de la comisión se mantienen en el cargo mientras cuenten con apoyo político.

c) En cuanto sus funciones. La Comisión Nacional de Bioética de Chile, de acuerdo con el proyecto original, se concibió como asesora del presidente de la República. Sin embargo, el texto definitivo (artículo 16 de la Ley $\mathrm{N}^{\circ} 20.120$ ) dispone que entre sus funciones debe asesorar a los distintos poderes del Estado en los asuntos éticos que se presenten como producto de los avances científicos y tecnológicos en biomedicina; en las materias relacionada con la investigación científica biomédica en seres humanos, recomendando la dictación, modificación y supresión de las normas que la regulen. Las resoluciones o acuerdos de la comisión se adoptan por simple mayoría, no obstante lo cual deberán hacerse constar las diferencias producidas en su seno y la posición de minoría.

Para llevar a efecto sus funciones, la Comisión cuenta con una secretaría ejecutiva que coordina su funcionamiento y canaliza los acuerdos que ella adopte, la que se estructurará en el Ministerio de Salud con el personal que al efecto se le asigne. El énfasis está puesto en que se trata de una comisión "asesora" de los poderes del Estado y como tal debe dar orientaciones y recomendaciones cuando un poder del Estado lo solicite. Sus recomendaciones no son vinculantes.

d) En cuanto a las observaciones. Se puede observar más solidez y legitimidad respecto del nombramiento de los integrantes en CONBIOÉTICA (en la que solo el ejecutivo designa) y CNBI (en la que nomina el presidente del Consiglio dei Ministri); en el caso nacional participan dos poderes del Estado (ejecutivo y legislativo), lo que se ha demostrado efectivo en la cultura nacional para representar diferentes sensibilidades en la integración de otros órganos colegiados.

En lo relativo a la dependencia de la CNB, ella se ubica en la estructura del Ministerio de Salud, que de suyo es una secretaría con dos subsecretarias y con muchas funciones. Sin embargo, consideramos que, por la importancia de la Comisión Nacional y por sus funciones asesoras de todos los poderes del Estado, su dependencia debe ser directa del presidente de la República y no del Ministerio de Salud. Refuerza lo anterior la experiencia comparada, en que las comisiones nacionales se ocupan de las cuestiones bioéticas de biomedicina, y además de temas vinculados a la ecología, medio ambiente, animales, etc.

Se presenta una observación de interpretación con relación a sus funciones: ¿qué se debe entender como "asuntos éticos que se presenten como producto de los avances científicos y tecnológicos en biomedicina; en las materias relacionadas con la investigación científica biomédica en seres humanos"? La sola lectura devela un ámbito de atribuciones ilimitado. Se pudiera entender que se trata de "todos" los avances de la biomedicina. Asimismo, es posible plantear si se trata de los 
avances solo nacionales o también de los extranjeros con aplicación en Chile. Se está en presencia de una "aldea global", con mayor razón en el ámbito biomédico.

\section{Discusión}

1. Parece oportuno citar la Declaración Universal de Bioética y Derechos Humanos de la Unesco, en cuanto a que las decisiones relativas a las cuestiones éticas relacionadas con la medicina, las ciencias de la vida, y las tecnologías tendrán repercusiones en las personas, familias, grupos o comunidades y en la especie humana en su conjunto. Por ello son de gran importancia quién, cómo y por qué se adoptan estas resoluciones.

2. La función de las comisiones no es regular temas bioéticos - tarea propia del ejecutivo y del Congreso-, sino definir, discutir y esclarecer problemas vinculados a la bioética; estudiarlos en profundidad para luego proponer criterios y recomendaciones a las autoridades del Estado, como asimismo informar y ayudar a formar a la comunidad toda.

3. Las propuestas de las comisiones nacionales de Bioética no son vinculantes para los poderes del Estado. Esto demanda de los miembros humildad, constancia y habilidad argumentativa y comunicacional. El diálogo y la razón son sus armas, y reconocer el pluralismo cultural instalado en nuestra sociedad. Es fundamental que el estudio, reflexión, discusión, asesoría y adopción de estas decisiones estén en manos de personas de la más alta competencia y de órganos representativos del Estado de Derecho propio de una sociedad democrática.

4. Son las comisiones quienes deben proponer líneas de acción, para que los países asuman con solvencia y capacidad los retos del acelerado desarrollo tecnocientífico, la creciente degradación de la biodiversidad natural, el desconocimiento de los derechos fundamentales a un gran número de personas y la exclusión económica, social, étnica y política de sectores de la población especialmente vulnerables.

5. El inicio de CONBIOÉTICA se concreta mediante un dictamen del presidente de la nación;
CNBI se concreta por decreto de la Presidenza del Consiglio dei Ministri, que recoge indicaciones de la Asamblea Parlamentaria del Consejo de Europa, de una Recomendación del Parlamento Europeo y de una solicitud de la cámara de diputados, lo que habla de su legitimidad de origen y mayor o menor vinculación con principios democráticos. Naturalmente, la posibilidad de implementar las comisiones nacionales por ley emanada de un Estado de derecho favorece en mayor grado el diálogo, el razonamiento y el reconocimiento de la identidad país.

Con todo, en el caso de México la legitimidad de la comisión está dada por el reconocimiento nacional e internacional de un trabajo serio y competente, convirtiéndose en un referente en el ámbito latinoamericano.

6. No parece sano que en CONBIOÉTICA (y en ninguna comisión de su naturaleza), su decreto de creación no indique un perfil de los candidatos, futuros integrantes.

Si bien el decreto de creación de CNBI no describe el perfil de candidatos, en su tradición normativa los miembros han estado ligados al mundo universitario, incluso cuando fueron en su primera nominación autoridades públicas designadas por derecho propio, a saber: presidente del Consejo Nacional de Investigación, presidente del Consejo Superior de Sanidad, presidente del Colegio Médico y director del Instituto Superior de Sanidad. Lo anterior se puede explicar por la "imparcialidad" que declara el órgano como obligación de los miembros en el ejercicio de sus funciones y reconocimiento expreso de garantías constitucionales.

En el caso del CNBI, se puede cuestionar la omisión de periodos de nominación de sus miembros; no obstante, en la cultura italiana ha permitido desarrollar una sana comunicación intergeneracional entre los miembros de la comunidad científica, lo que se refleja claramente en su composición y en el desarrollo de las actividades internacionales, que evidencian el liderazgo constante de los mayores.

7. Al respecto, es interesante replicar algunas de las buenas prácticas de la Comisión mexicana, en- 
tre otras, ser un espacio de intercambio de ideas y proyectos sobre el tema, organizando en forma regular foros y seminarios para académicos y para profesionales sobre sus temas propios. Y rindiendo cuentas en forma clara y explícita de su accionar una vez al año.

8. Entre las buenas prácticas de la Comisión italiana destaca la estructura pedagógica, uniforme y científica de sus documentos; que sus normativas internas manifiesten intención de identificar la documentación y la realidad social. Además, el diálogo y la discusión que se da en este órgano quedan registrados en su documentación, la cual es asequible a la comunidad nacional e internacional a través de su página web.

Cabe destacar también que es uno de los pocos comités nacionales de Europa en que los miembros ejercen su función ad honorem, como servicio gratuito al Estado, y en este sentido existe similitud a la situación chilena.

9. Se puede esperar que el corpus de normas consideradas en el seno de la CNB Chile no sea una importación y aplicación directa de las CIOMS5. En necesario considerar siempre la cultura, el derecho y la realidad nacional.

10. La CBN Chile debe asumir grandes desafíos. Es fundamental para su legitimación que sus integrantes sean personas de reconocido valor $y$ su trabajo sea de gran magnitud y profundidad. Cuando esta comisión se constituya, deberá proceder a dictar un reglamento para su organización y funcionamiento. En este cuerpo normativo se debe establecer la conformación de la agenda de trabajo para fijar prioridades, fechas y formas de reunión. Además de la participación y asesorías de expertos en la emisión de opiniones consultivas.

11. El real peso de esta nueva comisión lo darán sus recomendaciones éticas debidamente justificadas, con argumentos sustantivos, y también sus legítimos disensos fruto de un intercambio libre y racional entre sus integrantes. El debate en su seno debe ser público, con rigor lógico y epistemológico.

12. Es urgente y necesario promover una cultu-

${ }^{5}$ CIOMS, Council for International Organizations of Medical Science. Disponible en www.cioms.ch (30.04.2013). ra dialogante en todos los niveles de la sociedad chilena —escuelas, colegios y universidades-, para enfrentar los dilemas éticos resultantes del avance científico y tecnológico en las diferentes disciplinas de las ciencias de la vida, así como su impacto sobre la existencia en todos los seres vivos y el ecosistema.

13. La bioética en Chile, si quiere seguir lineamientos internacionales, debe considerar la experiencia de prácticas positivas de otros países de origen latino. No debiera seguir enclaustrada en las universidades y centros de investigación, debe ser capaz de tomar "la plaza pública" con cursos, foros y debates en los diferentes círculos sociales y medios de comunicación, ya que los temas de esta nueva disciplina involucran y demandan la atención de todas las personas. 


\section{Referencias}

1. Pacheco M. Teoría General del Derecho. Santiago de Chile: Editorial Jurídica de Chile: 1990.

2. Organización de las Naciones Unidas para la Educación, Ciencia y Cultura. Ciencias sociales y humanas, Asistencia a los Comités de Bioética, Objetivos, Comité Nacional de Bioética. Disponible en http://www.unesco.org/new/es/socialand-human-sciences/themes/bioethics/assisting-bioethics-committees/objective/national-bioethics-committees/ (07.05.2013).

3. Ministerio Educación Chile, Conicyt. Declaración universal sobre Bioética y Derechos Humanos. Disponible en http:// www.conicyt.cl/fonis/files/2013/03/Declaraci\%C3\%B3n-universal-sobre-Bio\%C3\%A9tica-y-Derechos-Humanos.pdf (07.05.2013).

4. Presidente Constitucional de los Estados Unidos Mexicanos 2005. D. O.F. 7 (2005), Crea la Comisión Nacional de Bioética. Disponible en http://www.paho.org/hq/index.php?option=com docman\&task=doc view\&gid=21389\&Itemid= (18.08.2013).

5. Presidenza dei Consiglio dei Ministri. Comitato Nazionale per la Bioetica. Disponible en http://www.governo.it/bioetica/normativa/decreto istitutivo.pdf (10.08.2013).

6. Congreso Nacional República de Chile. Ley No 20120 sobre investigación científica en el ser humano, su genoma, y prohibe la clonación humana. Disponible en http://www.leychile.cl/Navegar?idNorma=253478 (15.08.2013).

7. León FJ. Las Comisiones Nacionales de Bioética en Latinoamérica. Disponible en www.bioeticachile.cl/felaibe/documentos/libros/congreso (10.05.2013).

8. Universidad de Barcelona, Observatorio Bioética y Derecho. El Informe Belmont. Principios y guias éticos para la protección de los sujetos humanos de investigación. Disponible en http://www.pcb.ub.edu/bioeticaidret/archivos/norm/InformeBelmont.pdf (07.05.2013).

9. Casas M, Villela J, Comisiones Nacionales de Bioética en Latinoamérica. Desarrollo y oportunidades. Disponible en http:// www.bioeticachile.cl/felaibe/documentos/libros/congreso/FELAIBE\%20Actas.pdf (12.08.2013)

10. Serrano V, editor. Ética y globalización. Cosmopolitismo, responsabilidad y diferencia en un mundo global. Madrid: Biblioteca Nueva; 2004.

11. Presidenza dei Consiglio dei Ministri, Comitato Nazionale per la Bioetica. Linee Guida. Disponible en http://www. governo.it/bioetica/normativa/linee guida.pdf (19.08.2013).

12. Presidenza dei Consiglio dei Ministri, Comitato Nazionale per la Bioetica. Composizioni Comitato Nazionale per la Bioetica (1990 - 2013). Disponible en http://www.governo.it/bioetica/composizione cnb/index.html (12.08.2013)

13. Presidenza dei Consiglio dei Ministri, Comitato Nazionale per la Bioetica. Regolamento Interno. Disponible en http:// www.governo.it/bioetica/normativa/Regolamento.pdf (13.08.2013).

14. Assemblea Costituente. Costituzione della Reppublica Italiana (22.12.1947). Disponible en http://www.governo.it/Governo/Costituzione/CostituzioneRepubblicaItaliana.pdf (12.08.2013).

15. CONBIOÉTICA. Informe de rendición de cuentas de la Administración Pública Federal 2006-2012. Disponible en http://www.conbioetica-mexico.salud.gob.mx/descargas/pdf/ptrc/irc_2012.pdf. (30.04.2013).

16. Presidenza dei Consiglio dei Ministri, Comitato Nazionale per la Bioetica. Le attivitá a livello nazionale. Disponible en http://www.governo.it/bioetica/attivita 2011 2012.pdf (01.08.2013).

17. Congreso Nacional República de Chile. Historia de la Ley No 20120 sobre investigación cientifica en el ser humano, su genoma, y prohibe la clonación humana. Disponible en http://www.leychile.cl/Navegar?idNorma=253478 (15.08.2013).

18. Rodríguez E, Lolas F. Comisiones Nacionales de Bioética y Organismos Nacionales de Desarrollo de Ciencia y Tecnología en Latinoamerica. Una reflexión. Disponible en www.uchile.cl/download.jsd (21.05.2013).

Recibido: 21 de agosto de 2013

Aceptado: 29 de octubre de 2013 